\title{
Bulk experimental evidence of half-metallic ferromagnetism in doped manganites
}

\author{
Guo-meng Zhao and H. Keller \\ Physik-Institut der Universität Zürich, CH-8057 Zürich, Switzerland
}

\begin{abstract}
We report precise measurements and quantitative data analysis on the low-temperature resistivity of several ferromagnetic manganite films. We clearly show that there exists a $T^{4.5}$ term in lowtemperature resistivity, and that this term is in quantitative agreement with the quantum theory of two-magnon scattering for half metallic ferromagnets. Our present results provide the first bulk experimental evidence of half-metallic ferromagnetism in doped manganites.
\end{abstract}

The concept of half-metallic ferromagnets was first introduced by de Groot et al. in 1983 [1]. Half-metallic ferromagnets are characterized by completely spin-polarized electronic density of states at the Fermi level, that is, the majority spin channel is metallic while the Fermi energy falls in a band gap in the minority spin density of states. Such a novel physical property makes the materials very promising in technological applications such as single-spin electron sources and high-efficiency magnetic sensors. The half-metallic feature has been predicted for $\mathrm{CrO}_{2}$ [2]. However, spin-resolved photoemission measurement on the material has not confirmed this prediction [3]. On the other hand, the local density approximation (LDA) band-structure calculation on $\mathrm{La}_{2 / 3} \mathrm{Ca}_{1 / 3} \mathrm{MnO}_{3}$ [4] has shown an electronic structure of a nearly half-metallic ferromagnet (i.e., the majority spin channel is metallic while the Fermi energy lies within a band edge of the minority spin channel). Spinresolved photoemission study on a $\mathrm{La}_{0.7} \mathrm{Sr}_{0.3} \mathrm{MnO}_{3}$ film has demonstrated an electronic structure with spin polarization of nearly $100 \%$ at Fermi level, which possibly

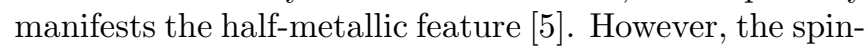
resolved tunneling measurements showed 54\% (Ref. [6]) and $81 \%$ (Ref. [7]) polarization of conduction electrons in $\mathrm{La}_{0.67} \mathrm{Sr}_{0.33} \mathrm{MnO}_{3}$. Since both photoemission and tunneling experiments are rather surface sensitive, the controversial conclusions drawn from these experiments are not so surprising. One needs to look for bulk sensitive experiments to unambiguously demonstrate whether the doped manganites are truly half-metallic ferromagnets or not. The clarification of this issue can place an essential constraint on the future prospect of this material in technological applications.

Since the Fermi level only crosses the majority spin bands in half-metallic ferromagnets, the emission or absorption of single magnon is forbidden, because the carriers have no conducting minority states at low energy to spin-flip scatter into. Therefore, the $T^{2}$ temperaturedependent term in the low-temperature resistivity due to single-magnon scatter [8] should be absent. Instead, twomagnon scattering is allowed, and leads to a $T^{4.5}$ temperature dependence in resistivity, as predicted by Kubo and Ohata [9]. Since this $T^{4.5}$ term should be rather small, it is difficult to identify this term if the other contributions to the resistivity are dominant. Nevertheless, if one can unambiguously identify the $T^{4.5}$ term, one provides bulk experimental evidence for the half-metallic nature. In this letter we not only show that there indeed exists the $T^{4.5}$ term in several manganite ferromagnets, but also demonstrate that the coefficient of the $T^{4.5}$ term is in quantitative agreement with the quantum theory of twomagnon scattering [9].

In half-metallic ferromagnets, the low-temperature resistivity due to two-magnon scattering was found to be $\rho_{K O}=A T^{4.5}$ (Ref. [9]). The coefficient $A$ has an analytical expression in the case of a simple parabolic conduction band (occupied by single-spin holes) [9]. In terms of the hole density per cell $n$, the average spin stiffness $\bar{D}$, and the effective hopping integral $t^{*}$, the coefficient $A$ can be written as [9]

$$
\begin{aligned}
A= & \left(\frac{3 a \hbar}{32 \pi e^{2}}\right)(2-n / 2)^{-2}\left(6 \pi^{2} n\right)^{5 / 3}\left(2.52+0.0017 \frac{\bar{D}}{a^{2} t^{*}}\right) \\
& \left\{\frac{a^{2} k_{B}}{\bar{D}\left(6 \pi^{2}\right)^{2 / 3}\left(0.5^{2 / 3}-n^{2 / 3}\right)}\right\}^{9 / 2} .
\end{aligned}
$$

Here we have used the relations: $a k_{F}=\left(6 \pi^{2} n\right)^{1 / 3}$ (where $\hbar k_{F}$ is the Fermi momentum, and $a$ is the lattice constant); $E_{F}=t^{*}\left(6 \pi^{2}\right)^{2 / 3}\left(0.5^{2 / 3}-n^{2 / 3}\right.$ ) (where the Fermi energy $E_{F}$ is measured from the band center); the effective spin $S^{*}=2-n / 2$. The value of $t^{*}$ can be estimated to be about $40 \mathrm{meV}$ from the measured effective plasma frequency $\hbar \Omega_{p}^{*}=1.1 \mathrm{eV}$ and $n \sim 0.3$ in $\mathrm{La}_{0.7} \mathrm{Ca}_{0.3} \mathrm{MnO}_{3}$ 10]. In ferromagnetic manganites, $\bar{D}$ is about $100 \mathrm{meV}$ $\overline{\mathrm{A}}^{2}$ (see below), so the term $0.0017 \bar{D} / a^{2} t^{*}<<2.52$, and can be dropped out in Eq. 1. Then there are two parameters $n$ and $\bar{D}$ that determine the magnitude of $A$. In doped manganites, $n$ should be approximately equal to the doping level $x$, as it is the case in $\mathrm{La}_{1-x} \mathrm{Sr}_{x} \mathrm{MnO}_{3}$ system (see below). The average spin stiffness $\bar{D}$ should be close to the long-wave spin stiffness $D(0)$ if there is negligible magnon softening near the zone boundary.

It has recently been shown [11] that the dominant contribution to the low-temperature resistivity is due to scattering from a soft optical phonon mode, which gives a term proportional to $\omega_{s} / \sinh ^{2}\left(\hbar \omega_{s} / 2 k_{B} T\right)$, where $\omega_{s}$ is the frequency of a soft optical mode. If we include a 
possible contribution from two-magnon scattering [9], or from acoustic-phonon scattering [8], the temperature dependent part of the resistivity can be generally expressed as

$$
\rho(T)-\rho_{o}=A T^{\alpha}+B \omega_{s} / \sinh ^{2}\left(\hbar \omega_{s} / 2 k_{B} T\right),
$$

where $\rho_{o}$ is the residual resistivity; $A$ and $B$ are temperature independent coefficients. The power $\alpha=4.5$ if the major contribution is from two-magnon scattering, while $\alpha=5$ if the main contribution is due to acoustic phonon scattering. We can make a distinction between the two cases. For acoustic phonon scattering, the coefficient $A$ should be independent of the applied magnetic field if $m^{*} / n$ or $\rho_{o}$ does not depend on the field (where $m^{*}$ is the effective mass of carriers). This is because $A \propto$ $\left(\frac{m^{*}}{n}\right)^{2}\left(\theta_{D}\right)^{-5}$ in this case, where $\theta_{D}$ is the Debye temperature [8]. For two-magnon scattering, a magnetic field induces a gap in spin-wave excitations, so that $A$ should decrease with increasing magnetic field.

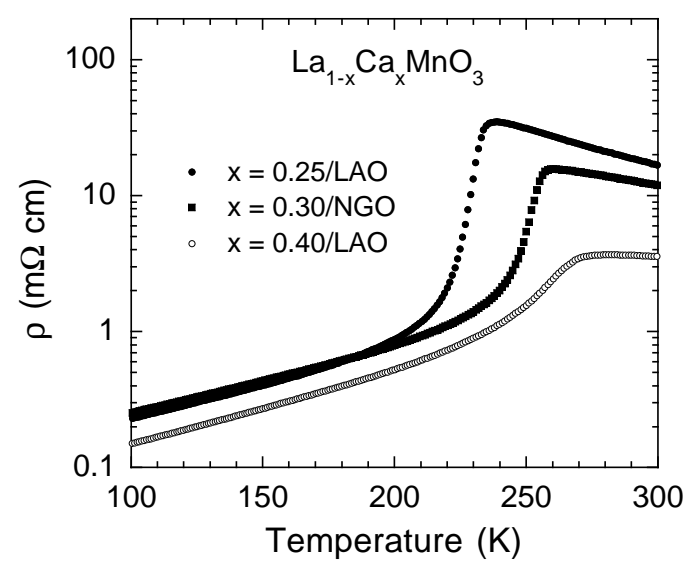

FIG. 1. The resistivity of the films $\mathrm{La}_{1-x} \mathrm{Ca}_{x} \mathrm{MnO}_{3}$ over $100-300 \mathrm{~K}$. As $x$ increases, the metal-insulator crossover temperature increases.

Epitaxial thin films of $\mathrm{La}_{1-x} \mathrm{Ca}_{x} \mathrm{MnO}_{3}$ were grown on $<100>\mathrm{LaAlO}_{3}(\mathrm{LAO})$ or $\mathrm{NdGaO}_{3}(\mathrm{NGO})$ single crystal substrates by pulsed laser deposition using a $\mathrm{KrF}$ excimer laser [12]. The films were post annealed in 1 bar oxygen at $940{ }^{\circ} \mathrm{C}$ for $10 \mathrm{~h}$. The resistivity was measured using the van der Pauw technique, and the contacts were made by silver paste. The measurements were carried out in a Quantum Design measuring system.

Fig. 1 shows the temperature dependence of the resistivity for the films of $\mathrm{La}_{1-x} \mathrm{Ca}_{x} \mathrm{MnO}_{3}$ over $100-300 \mathrm{~K}$. As $x$ increases the metal-insulator crossover temperature increases. The Curie temperature $T_{C}$ should coincide with a temperature where $d \ln \rho / d T$ exhibits a maximum. The $T_{C}$ values with this definition are listed in Table I.

In Fig. 2, we show the temperature-dependent part of the resistivity at low temperatures for $\mathrm{La}_{0.75} \mathrm{Ca}_{0.25} \mathrm{MnO}_{3}$ film measured in zero magnetic field (a), and in a magnetic field of $4 \mathrm{~T}$ (b). We fit the data in zero field by Eq. 2 using three fitting parameters: $A, B$ and $\omega_{s}$, and a fixed $\alpha=5$. Meanwhile, we fit the data in $4 \mathrm{~T}$ magnetic field with two fitting parameters: $A$ and $B$, and with fixed $\omega_{s}$ and $\alpha$ which are the same as those in zero field case. One can see that the fits are very good in both cases. Note that we have excluded the data above $100 \mathrm{~K}$ in the fitting since $n / m^{*}$ above $100 \mathrm{~K}$ becomes temperature dependent [10].

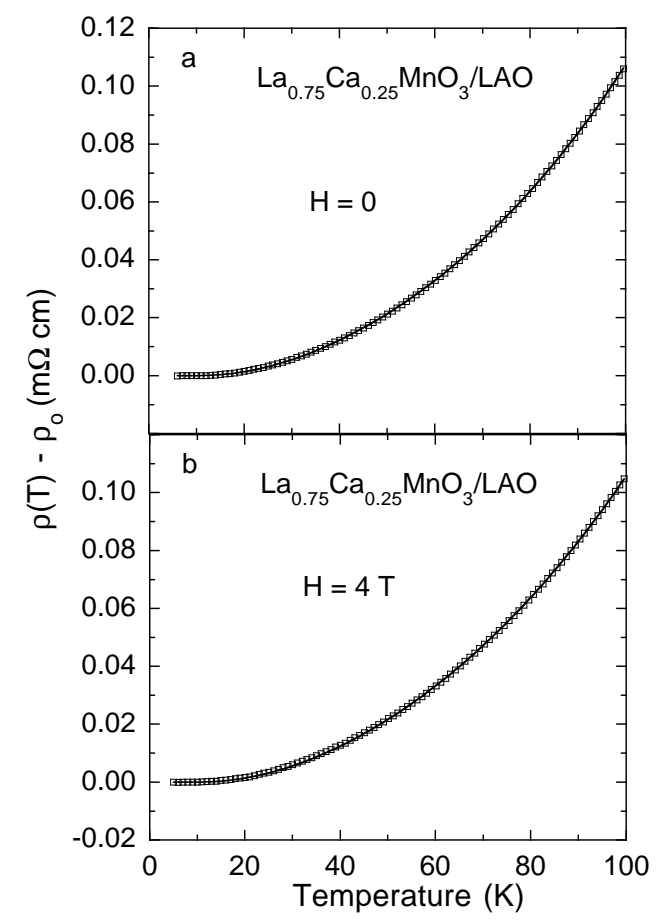

FIG. 2. The temperature-dependent part of the resistivity at low temperatures for the film of $\mathrm{La}_{0.75} \mathrm{Ca}_{0.25} \mathrm{MnO}_{3}$ measured in zero magnetic field (a), and in $4 \mathrm{~T}$ magnetic field (b). The solid lines are the fitted curves by Eq. 2 with a fixed $\alpha=5$. The residual resistivity $\rho_{o}$ does not depend on the magnetic field within the experimental uncertainty.

An important finding from the fits is that the parameters $B$ and $\omega_{s}$ are independent of magnetic field, while the value of the parameter $A$ in $4 \mathrm{~T}$ magnetic field is smaller than that in zero magnetic field by a factor of 1.5. Such a strong field dependence of the coefficient $A$ is not expected from acoustic phonon scattering unless $m^{*} / n$ or $\rho_{o}$ also strongly depends on the field [8]. The fact that $\rho_{o}$ is independent of the magnetic field rules out the possibility that the $A T^{\alpha}$ term in Eq. 2 can arise from acoustic phonon scattering.

Fig. 3 shows the temperature-dependent part of the resistivity at low temperatures in zero magnetic field for $\mathrm{La}_{1-x} \mathrm{Ca}_{x} \mathrm{MnO}_{3}$ films with different $x$. The solid lines are the fitted curves by Eq. 2 with three fitting parameters: $A, B$ and $\omega_{s}$, and with a fixed $\alpha=4.5$. The values of the 
fitting parameter $A$ are summarized in Table I. The $\hbar \omega_{s}$ value is $6.41 \pm 0.03 \mathrm{meV}$ for $x=0.25,7.40 \pm 0.06 \mathrm{meV}$ for $x=0.30$, and $6.90 \pm 0.06 \mathrm{meV}$ for $x=0.40$. The doping dependence of $\omega_{s}$ is very simialr to that of $\theta_{D}$ found for $\mathrm{La}_{1-x} \mathrm{Sr}_{x} \mathrm{MnO}_{3}$ system [13]. The absolute values of $\hbar \omega_{s}$ are very close to the phonon energy $(6 \mathrm{meV})$ of the rotational vibrations of the oxygen octahedra in a similar perovskite $\mathrm{Ba}\left(\mathrm{Pb}_{0.75} \mathrm{Bi}_{0.25}\right) \mathrm{O}_{3}$ [14]. This rotational mode is shown to be strongly coupled to conduction electrons by tunneling experiment [14].
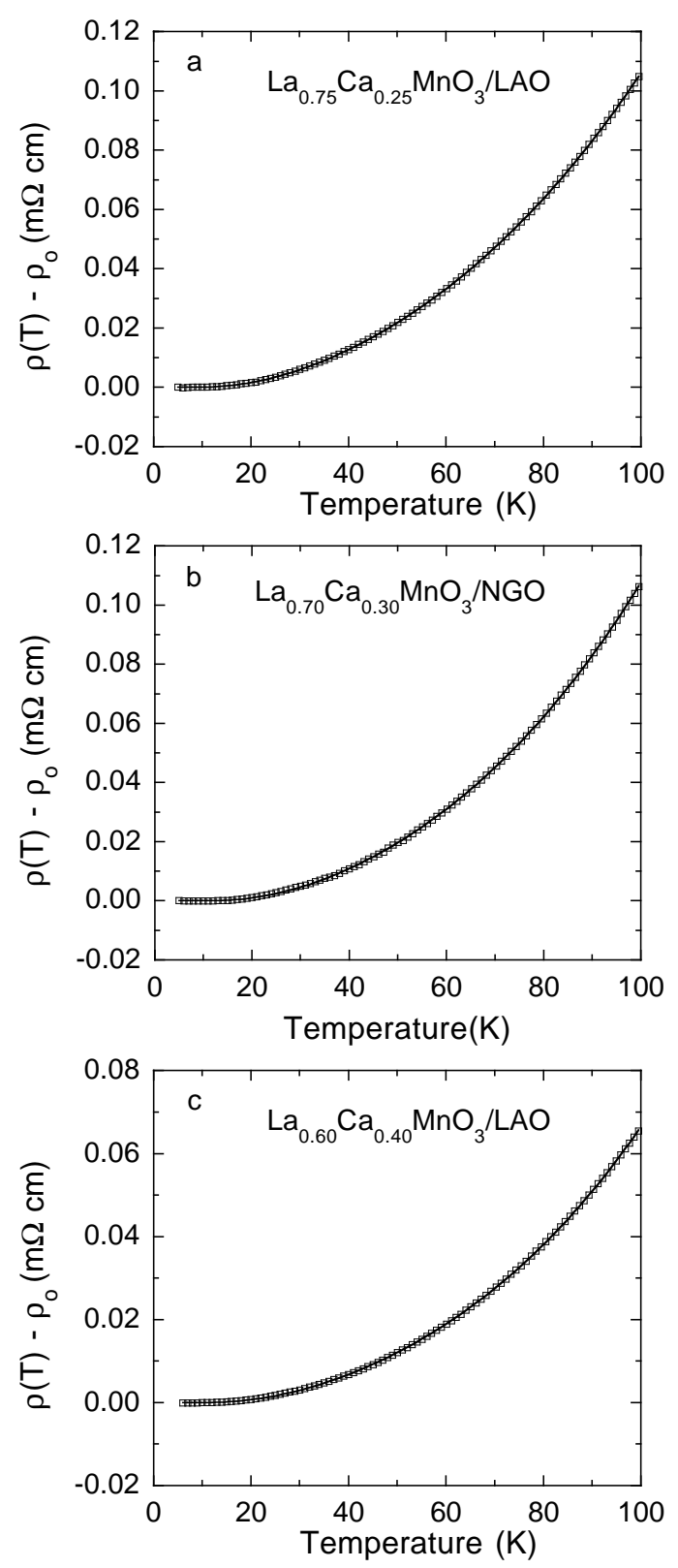

FIG. 3. The temperature-dependent part of the resistivity at low temperatures for the $\mathrm{La}_{1-x} \mathrm{Ca}_{x} \mathrm{MnO}_{3}$ films with different $x$. The solid lines are fitted curves by Eq. 2 with a fixed $\alpha=4.5$.

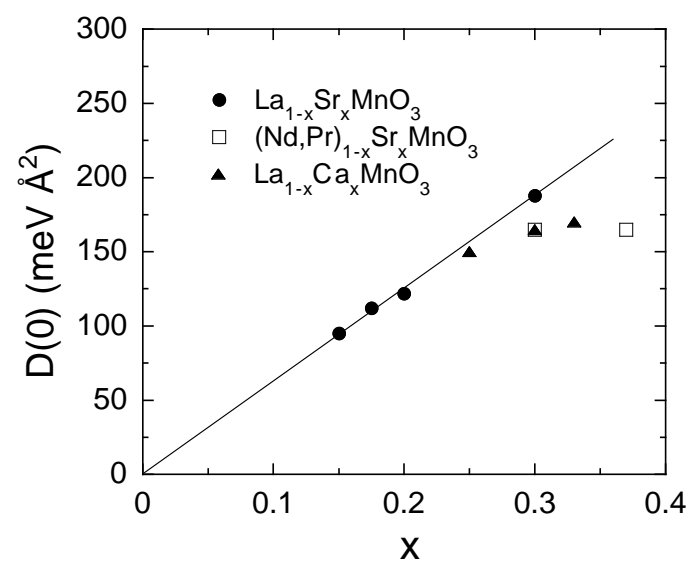

FIG. 4. The long-wave spin stiffness $D(0)$ as a function of $x$ for different manganite systems. The data of $\mathrm{La}_{1-x} \mathrm{Sr}_{x} \mathrm{MnO}_{3}$ are taken from Ref. 18, 19. The data of $\mathrm{La}_{1-x} \mathrm{Ca}_{x} \mathrm{MnO}_{3}$ are from Ref. 20 21]. The data of (Nd,Pr $)_{1-x} \mathrm{Sr}_{x} \mathrm{MnO}_{3}$ are taken from Ref. 22.

In order to further confirm that the $T^{4.5}$ term indeed originates from two-magnon scattering, it is neccessary to show that the values of the coefficient $A$ in Table I should be in quantitative agreement with Eq. 1 . Since the $A$ value in Eq. 1 is sensitive to the hole density $n$, it is essential to determine the $n$ value more reliably. Roughly speaking, one might expect that $n \simeq x$. Since doped holes mainly reside on the oxygen sites 15, 16, each oxygen hole will produce a ferromagnetically coupled bond with an exchange energy $J \sim t_{p d}^{4} / \Delta^{3}$, where $t_{p d}$ is a hybridization matrix element between the $d$ and $p$ orbitals, and $\Delta$ is a charge transfer gap [17]. Then one can readily show that the long-wave spin stiffness $D(0) \propto n$. In Fig. 4, we plot $D(0)$ as a function of $x$ for different manganite systems. For $\mathrm{La}_{1-x} \mathrm{Sr}_{x} \mathrm{MnO}_{3}$ system, the linear relation between $D(0)$ and $x$ holds up to $x=0.3$, implying that $n=x$ for $0.15 \leq x \leq 0.3$. For $\mathrm{La}_{1-x} \mathrm{Ca}_{x} \mathrm{MnO}_{3}$ and (Nd,Pr) $)_{1-x} \mathrm{Sr}_{x} \mathrm{MnO}_{3}$ systems, $D(0)$ starts to deviate

TABLE I. The summary of the fitting parameter $A$, the measured $T_{C}$, the estimated hole density per cell $n$, and the calculated $\bar{D} / k_{B} T_{C}$ for $\mathrm{La}_{1-x} \mathrm{Ca}_{x} \mathrm{MnO}_{3}$. The uncertainty in $T_{C}$ is $\pm 1 \mathrm{~K}$. The measured values of $T_{C}$ and $D(0) / k_{B} T_{C}$ from neutron scattering for $\mathrm{La}_{0.7} \mathrm{Sr}_{0.3} \mathrm{MnO}_{3}$ (LSMO30) 23] are also included in the last row.

\begin{tabular}{|c|c|c|c|c|c|}
\hline $\begin{array}{l}\text { Compounds } \\
x\end{array}$ & $\begin{array}{l}T_{C} \\
(\mathrm{~K})\end{array}$ & $n$ & $\begin{array}{c}A \\
\left(\mathrm{~m} \Omega \mathrm{cm} / \mathrm{K}^{4.5}\right)\end{array}$ & $\frac{\bar{D}}{k_{B} T_{C}}$ & $\begin{array}{c}\frac{D(0)}{k_{B} T_{C}} \\
\left(\AA^{2}\right)\end{array}$ \\
\hline$\overline{0.25(\mathrm{H}=0)}$ & 232 & 0.24 & $1.20(2) \times 10^{-11}$ & 5.97 & \\
\hline $0.25(\mathrm{H}=4 \mathrm{~T})$ & & & $0.82(2) \times 10^{-11}$ & & \\
\hline 0.30 & 253 & 0.26 & $1.70(3) \times 10^{-11}$ & 5.71 & \\
\hline 0.40 & 262 & 0.26 & $1.27(3) \times 10^{-11}$ & 5.88 & \\
\hline LSMO30 & 378 & & & & $5.8(2)$ \\
\hline
\end{tabular}


from the linear relation above $x=0.2$ and remains a constant for $0.30 \leq x \leq 0.40$. From the linear relation between $n$ and $D(0)$, we find $n=0.24$ for $x=0.25, n=$ 0.26 for $x=0.30$ and 0.40 .

Now we can calculate the average spin stiffness $\bar{D}$ using Eq. 1 and the $A$ values listed in Table I. The calculated $\bar{D} / k_{B} T_{C}$ values for three manganite compounds are summarized in Table I. It is remarkable that the $\bar{D} / k_{B} T_{C}$ values deduced from the resistivity data are very close to the $D(0) / k_{B} T_{C}$ value $\left(5.8 \pm 0.2 \AA^{2}\right)$ for $\mathrm{La}_{0.7} \mathrm{Sr}_{0.3} \mathrm{MnO}_{3}$ from neutron scattering [23]. Since the magnon softening near the zone boundary is negligible when the $T_{C}$ is higher than $350 \mathrm{~K}$ [24], there should a negligible magnon softening in the compound $\mathrm{La}_{0.7} \mathrm{Sr}_{0.3} \mathrm{MnO}_{3}$ with the highest $T_{C}=378 \mathrm{~K}$. In this case, one should expect that $\bar{D} \simeq$ $D(0)$. On the other hand, $\bar{D}<D(0)$ if there is a magnon softening near the zone boundary as the case of low $T_{C}$ materials [24]. In any cases, one might expect that the average $\bar{D}$ should be proportional to $T_{C}$ so that $\bar{D} / k_{B} T_{C}$ is a univeral constant in the manganite system. This has indeed been verified by the result shown in Table I. Therefore, our present results provide a quantitative confirmation for the quantum theory of two-magnon scattering.

Since the quantum theory of two-magnon scattering is valid only for half-metallic ferromagnets, the quantitative proof for the theory in the doped manganites gives bulk experimental evidence that the ferromagnetic manganites are indeed half-metallic materials. We are not aware of any other materials which have been confirmed to be half-metallic ferromagnets by bulk-sensitive experiments. The unique half-metallic nature only found in these doped manganites makes them one of the most important and useful materials in future technological applications.

In summary, we report precise measurements and quantitative data analysis on the low-temperature resistivity of several ferromagnetic manganite films. We show that there exists a $T^{4.5}$ term in low-temperature resistivity, and that this term is in quantitative agreement with the quantum theory of two-magnon scattering for half metallic ferromagnets. Our present results provide the first bulk experimental evidence of half-metallic ferromagnetism in doped manganites.

Acknowledgement: We would like to thank W. Prellier and D. J. Kang for providing high-quality manganite films. The work was supported by the Swiss National Science Foundation.

[1] R. A. de Groot, F. M. Müller, P. G. van Engen, and K. H. J. Buschow, Phys. Rev. Lett. 50, 2024 (1983).
[2] K. Schwarz, J. Phys. F 16, L211 (1986).

[3] K. P. Kämper, W. Schmitt, G. Güntherodt, R. J. Gambin, and R. Ruf, Phys. Rev. Lett. 59, 2788 (1988).

[4] W. E. Pickett and D. J. Singh, Phys. Rev. B 53, 1146 (1996).

[5] J. H. Park, E. Vescovo, H.-J. Kim, C. Kwon, R. Ramesh, and T. Venkatesan, Nature (London) 392, 794 (1998).

[6] Y. Lu et al., Phys. Rev. B 54, R8357 (1996).

[7] J. Z. Sun, L. Krusin-Elbaum, P. R. Duncombe, A. Gupta, and R. B. Laibowwitz, Appl. Phys. Lett. 70, 1769 (1997).

[8] I. Mannari, Prog. Theor. Phys. 22, 335 (1959).

[9] K. Kubo and N. A. Ohata, J. Phys. Soc. Jpn. 33, 21 (1972).

[10] J. R. Simpson, H. D. Drew, V. N. Smolyninova, R. L. Greene, M. C. Robson, A. Biswas, and M. Rajeswari, Phys. Rev. B 60, R16 263 (1999).

[11] G. M. Zhao, V. Smolyaninova, W. Prellier, and H. Keller, Phys. Rev. Lett. 84, 6086 (2000).

[12] W. Prellier, M. Rajeswari, T. Venkatesan, and R. L. Greene, Appl. Phys. Lett. 75, 1446 (1999).

[13] T. Okuda, A. Asamitsu, Y. Tomioka, T. Kimura, Y. Taguchi, and Y. Tokura, Phys. Rev. Lett. 81, 3202 (1998).

[14] W. Reichardt, B. Batlogg, and J. P. Remeika, Physica B 135, 501 (1985).

[15] T. Saitoh, A. E. Bocquet, T. Mizokawa, H. Namatame, A. Fujimori, M. Abbate, Y. Takeda, and M. Takano, Phys. Rev. B 51, 13942 (1995).

[16] H. L. Ju, H. C. Sohn, and K. M. Krishnan, Phys. Rev. Lett. 79, 3230 (1997).

[17] D. I. Khomskii and G. A. Sawatzky, Solid State Commun. 102, 87 (1997).

[18] K. Hirota, N. Kaneko, Y. Endoh, M. C. Martin, and G. Shirane, Physica B 237-238, 36 (1997).

[19] L. Vasiliu-Doloc, J. W. Lynn, A. H. Moudden, A. M. de Leon-Guevara, and A. Revcolevschi, Phys. Rev. B 58, 14913 (1998).

[20] P. Dai, H. Y. Hwang, J. Zhang, J. A. Fernandez-Baca, S.-W. Cheong, C. Kloc, Y. Tomioka, and Y. Tokura, Phys. Rev. B 61, 9553 (2000); D(0) = $150 \mathrm{meV} \AA^{2}$ for $\mathrm{La}_{0.75} \mathrm{Ca}_{0.25} \mathrm{MnO}_{3}$ (unpublished data).

[21] J. W. Lynn, R. W. Erwin, J. A. Borchers, Q. Huang, A. Santoro, J. L. Peng, and Z. Y. Li, Phys. Rev. Lett. 76, 4046 (1996).

[22] J. A. Fernandez-Baca, P. Dai, H. Y. Hwang, C. Kloc, and S.-W. Cheong, Phys. Rev. Lett. 80, 4012 (1998).

[23] M. C. Martin, G. Shirane, Y. Endoh, K. Hirota, Y. Moritomo, and Y. Tokura, Phys. Rev. B 53, 14285 (1996).

[24] H. Y. Hwang, P. Dai, S.-W. Cheong, G. Aeppli, D. A. Tennant, and H. A. Mook, Phys. Rev. Lett. 80, 1316 (1998). 